\title{
L'AROME DU BEURRE ET SON ORIGINE
}

\author{
par C. LIND.
}

L'arôme du beurre provient, en grande partie, des substances volatiles formées pendant la maturation de la crème et qui se dissolvent aisément dans la matière grasse. Le beurre qui est fabriqué avec de la crème douce n'a qu'un arôme très peu marqué.

Dans cet article, nous démontrerons la formation de ces substances pendant la maturation de la crème et nous chercherons à apprécier dans quelle mesure le beurre est capable de les dissoudre.

B. W. Hammer, du Collège d'Agriculture de i'Etat d'Iowa, a beaucoup travaillé sur la question de la formation des acides volatiles dans les cultures de ferments lactiques aux différentes phases de leur dévelop. pement. Voici la technique qu'il recommande pour mesurer la quantité de ces acides volatils :

250 gr. de cultures sont versés dans un grand ballon. On ajoute $15 \mathrm{em}^{3}$ d'acide sulfurique normal et l'on entraîne avec de la vapeur d'eau jusqu'à ce qu'un litre soit distillé. On fait le dosage titrimétrique avec une solution $\mathrm{N} / 10$ de soude et le nombre employé de $\mathrm{cm}^{3}$ de cette liqueur sodique sert de mesure aux acides volatils, en dépit du fait qu'on n'obtient pas par cet entraînement à la vapeur d'eau tous ces derniers.

Ceci est, en effet, assez clairement démontré par l'expérience suivante que j'ai réalisée :

250 gr. d'une culture dans le lait de ferments lactiques avec une acidité de 114 (1) sont distillés d'après la méthode de HAMMER, mais on recueille le liquide qui passe par portions de $100 \mathrm{~cm}^{3}$. Après la distillation d'un litre de liqueur, un autre litre est distillé, que l'on recueille par fractions de $250 \mathrm{~cm}_{8}$, qui sont titrées également.

Voici les chiffres que l'on obtient:

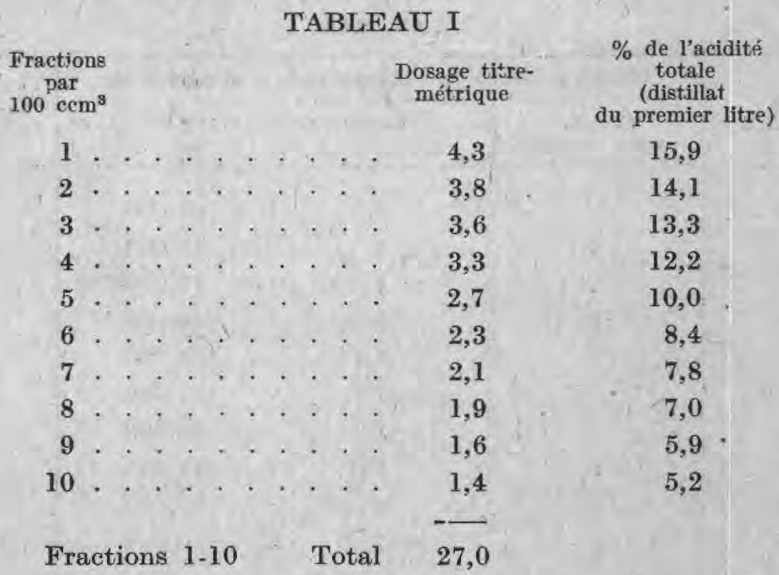

(1) L'acidité est mesurée par le nombre de $\mathrm{ccm}^{3}$ de soude $\frac{\mathrm{N}}{10}$ pour $100 \mathrm{ccm}^{3}$ de culture. 


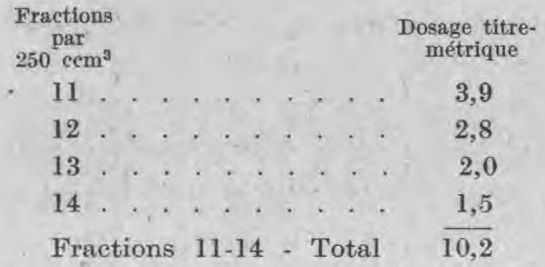

Il résulte de ce tableau que l'acidité des fractions recueillies va en diminuant; mais alors que l'acidité totale du premier litre recueilli est de 27 , il reste encore dans la liqueur une quantité assez considérable d'acides volatils, puisque l'acidité du second litre recueilli titre 10,2. Si la distillation est toujours exécutée de la même manière, il est permis de conclure que la quantité d'acides volatils recueillis dans le premier litre est à peu près proportionnelle à la quantité totale et, par conséquent, sa détermination peut servir de mesure aux acides volatils.

Il est connu, depuis les travaux de Hammer, V. STorch, F. W. J. Вовскноuт et $O$. de VRies, que de bons ferments lactiques sélectionnés pour l'acidification de la crème, contiennent non seulement des bactéries produisant de l'acide lactique, mais, à côté, des bactéries qui donnent de l'arôme, ce qui veut dire des acides volatils, mais peu d'acide lactique. C'est en symbiose avec les premières que celles-ci forment la plus grande quantité d'acides volatils.

HAMMER rapporte que de bonnes cultures bien mûres donnent un distillat qui, par titrimétrie, correspond à $30 / 40 \mathrm{~cm}^{3} \mathrm{~N} / 10$ d'acide normal. La quantité d'acides dépend d'ailleurs au plus haut degré de la maturation, de l'âge del la culture, de la température à laquelle elle pousse, etc. Une culture de Chr. Hansen qui s'est développée dans des conditions semblables à celles des expériences de HAMmer a donné un distillat titrant $38 \mathrm{~cm}^{3} \mathrm{~N} / 10$ d'acides volatils. HaMmer a démontré que la quantité d'acides volatils va croissant avec l'acidité totale de la culture, ce qui résulte du tableau suivant extrait de son travail.

\section{TABLEAU II}

Acidité totale et acides volatils d'une culture se développant à $21^{\circ} \mathrm{C}$.

Nombre

d'heures de

culture

15

16

17

18

19

20

$21^{*}$

22

39 pendant des temps différents.

$\begin{array}{cccc}\begin{array}{c}\% \text { d'acide } \\ \text { lactique }\end{array} & \begin{array}{c}\text { Acidité totale } \\ \text { Degré } \\ \text { d'acidité }\end{array} & \begin{array}{c}\text { Aridité du } \\ \text { distillat }\end{array} & \begin{array}{c}\% \text { de } \\ \text { l'acidité } \\ \text { totale }\end{array} \\ 0,68 & 76 & 7,5 & 3,9 \\ 0,76 & 84 & 10,0 & 4,7 \\ 0,81 & 90 & 11,9 & 5,3 \\ 0,84 & 93 & 15,2 & 6,5 \\ 0,86 & 96 & 17,1 & 7,2 \\ 0,88 & 98 & 21,5 & 8,8 \\ 0,91 & 101 & 26,0 & 10,2 \\ 0,93 & 103 & 28,3 & 11,0 \\ 1,10 & 122 & 34,5 & 11,3\end{array}$


L'acidité totale a été mesurée sur $100 \mathrm{~cm}^{3}$ de culture et le distillat a été obtenu avec $250 \mathrm{~cm}^{3}$, d'où la nécessité de faire une correction pour pouvoir comparer les deux valeurs. La dernière colonne nous montre que le taux des acides volatils s'élève relativement plus vite que l'acidité totale et qu'il est très faible pour des acidités totales peu élevées.

Des recherches que j'ai faites m'ont donné des résultats analogues toutes les fois que j'utilisais la méthode de HAMmer. Il m'est arrivé d'effectuer des distillations sans addition d'acide sulfurique ainsi qu'il est noté dans le tableau III ; les résultats obtenus ne diffèrent que d'une façon insignifiante de ceux que nous donnent des distillations exécutées sur le même échantillon en présence d'acide sulfurique. J'avais, en effet, l'intention de voir si la mise en liberté des acides volatils par l'acidité propre des cultures suffirait pour mes déterminations et $j$ 'ai vu, ainsi que je viens de le dire et en accord avec ce qu'avait trouvé V. STORCH, que la différence entre les deux méthodes de distillation était seulement très faible. J'ai cependant donné la préférence à la méthode qui utilise l'acide sulfurique.

Dans le tableau suivant, on voit que l'augmentation des acides volatils ne monte pas aussi régulièrement avec l'acidité totale, comme on a pu le constater dans le tableau précédent de Hammer. Je crois qu'il faut trouver l'explication de ce fait dans ce que mes cultures ont été faites dans des circonstances variables; or, nous savons que le rapport des acides volatils à l'acidité totale est relativement dépendant de ces eirconstances.

TABLEAU III

Titre des acides volatils.

$\begin{array}{ccc}\text { Acidité } & \begin{array}{c}\text { Avec acide } \\ \text { sulfurique }\end{array} & \begin{array}{c}\text { Sans acide } \\ \text { sulfurique }\end{array} \\ 14 & 3,2 & \\ 18 & 4,2 & \\ 19 & 3,5 & 11,6 \\ 45 & 8,3 & 21,0 \\ 90 & & \\ 92 & & 20,5 \\ 93 & 18,8 & 20,0 \\ 98 & & 24,0 \\ 102 & & 18,3 \\ 102 & 27,5 & 14,5-17,0 \\ 105 & 22,0 & \\ 106 & 18,7 & \\ 113 & 25,3 & \\ 114 & 27,0 & \end{array}$

Le résultat des expériences de Hammer et de mes propres expériences est que ce n'est qu'après que l'acidité totale des cultures a atteint un taux assez élevé qu'on voit se former des quantités considérables d'acides volatils. Ce qui 
veut dire, en d'autres termes, qu'on ne peut pas compter sur la formation d'un arome prononcé dans le beurre sans une acidification de la crème.

Des recherches nouvelles ont montré que, par la distillation, des quantités considérables d'acide lactique peuvent passer, ce qui n'était pas admis auparavant. Pour examiner dans quelle mesure ce fait pouvait influencer les expériences dont il est parlé, j'ai entrepris quelques distillations d'un lait pasteurisé additionné de quantités variables d'acide acétique normal et d'acide lactique normal, en employant comme précédemment $15 \mathrm{~cm}^{3}$ d'acide sulfurique pour $250 \mathrm{~cm}^{3}$ de lait.

TABLEAU IV

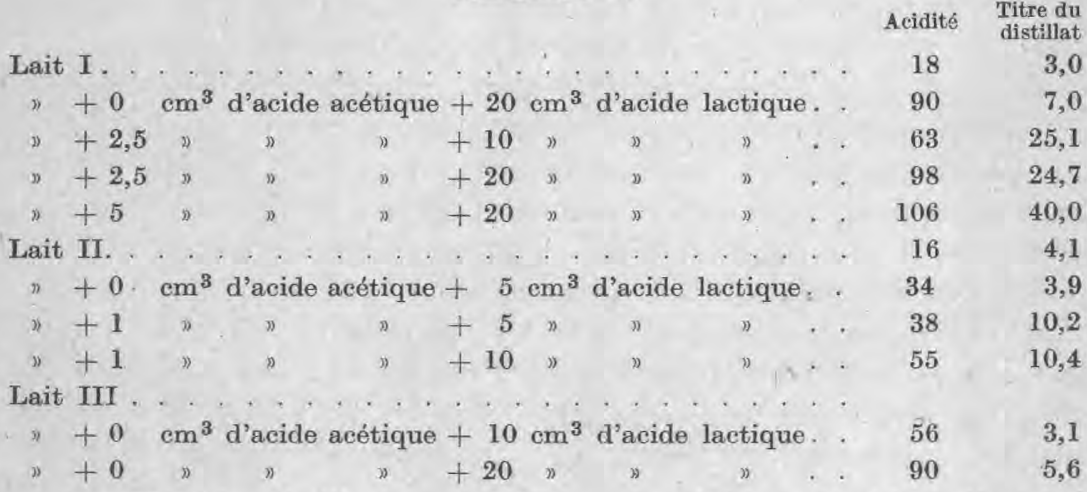

L'acidité du distillat du lait III sans addition d'acide sulfurique n'a pas été déterminée.

Le tableau IV nous montre que le distillat du lait sans addition préalable d'acide acétique ou d'acide lactique a un titre qui oscille de 3 à 4. Aussi, il est permis de conclure que dans le cas du lait III additionné de $10 \mathrm{~cm}^{3}$ d'acide lactique, comme dans le cas du lait II additionné de $5 \mathrm{~cm}^{3}$ de cet acide, ces additions n'ont pas influé sur le titre du distillat. Quand ce titre a varié, c'est uniquement du fait de l'acide acétique, ainsi qu'on peut s'en rendre compte pour une même quantité d'acide acétique, mais une addition de $20 \mathrm{~cm}^{3}$ d'acide lactique aux Laits I et III ont augmenté le titre du distillat. On peut augmenter la quantité d'acide lactique, celan'a pas d'influence sur le titre du distillat, sauf cependant dans les cas où les proportions d'acide lactique sont assez fortes. A.J. VIRTANEN a constaté que l'acide lactique en petites quantités distillait avec la vapeur d'eau.

En distillant $200 \mathrm{~cm}^{3}$ de lait frais après addition de $48 \mathrm{~cm}^{3}$ d'acide lactique normal, il a trouvé $1 \mathrm{~cm}^{3}$ de cet acide dans les premiers $800 \mathrm{~cm}^{3}$.

Les acides volatils distillent moins aisément quand ils sont dans le lait que lorsqu'il sont dans l'eau pure. $200 \mathrm{~cm}^{3}$ d'une culture de coccus producteur d'acide lactique, culture âgée de 12 jours, faite à $20^{\circ} \mathrm{C}$., ayant 113 d'acidité, furent distillés sous la vapeur d'eau par fractions de $400 \mathrm{~cm}^{3}$ jusqu'à ce qu'on obtienne un total de $2.400 \mathrm{~cm}^{3}$. Le titre 
de ces $2.400 \mathrm{~cm}^{8}$ était de 51,0 et il s'abaisse lorsqu'on en soustrait l'acide lactique distillé à 48,5 , soit $21,5 \%$ de l'acidité totale de la culture; eette valeur est plus grande que celle qui est indiquée dans le tableau de Hammer, colonne 5. Mais la eulture de Virtanen était plus 'âgée et probablement pas de la même espèce microbienne et en outre, il faut noter que VIRTANen a distillé des quantités beaucoup plus grandes, ce qui, d'après mes expériences, ne peut qu'accroître la différence de ses résultats avec ceux de Hammer.

Nous arrivons maintenant à considérer la question qui, au point de vue pratique, a une grande portée: Combien a matière grasse $d u$ beurre peut-elle absorber de ces acides volatils? C'est là un problème difficile à résoudre et, à mon avis, les expériences de laboratoire ne suffisent pas, du moins elles ne peuvent aider à éclaircir le problème.

La détermination des acides volatils dans le beurre est difficile.

Dans ce travail, je n'ai déterminé qu'une partie de ces acides volatils, eelle qui a passé dans les conditions analogues à celles auxquelles nous avons eu recours pour la distillation des cultures. Nous avons pris $100 \mathrm{gr}$. de beurre auxquels nous avons ajouté $150 \mathrm{~cm}^{3}$ d'eau et $15 \mathrm{~cm}$. d'acide sulfurique normal qu'on distille dans la vapeur d'eau jusqu'à ce qu'on recueille 1 litre et ce litre est titré avee de la soude N/10. Avant de parler de nos expériences, voyons celles des autres expérimentateurs Ferris, Readfield et North, qui ont utilisé la méthode suivante :

50 gr. de beurre $+5 \mathrm{~cm}^{3}$ d'une solution d'acide phosphorique à $25 \%+100 \mathrm{~cm}^{3}$ d'eau sont distillés en présence de la vapeur d'eau, de façon que l'on recueille $100 \mathrm{~cm}^{3}$ pendant 10 minutes. Le distillat est ensuite titré avec de la soude $\mathrm{N} / 10$.

Nécessairement, les résultats obtenus par ces auteurs sont plus faibles que les miens, puisqu'ils ont recueilli des quantités inférieures, et par suite ils ne peuvent pas être comparés à ceux que j'ai obtenus. Toutefois, la conclusion de leurs expériences est de même ordre que la mienne, à savoir que la quantité d'acides volatils trouvée dans le beurre s'élève en même temps que l'acidification de la crème qui a servi à le préparer.

Dans leur tableau I, le titre du beurre provenant de crème fraîche était de 0,2 . Si la crème avait subi une faible acidification, le titre était de 0,4 , avec un maximum de 0,7 . (Les titres sont indiqués pour 100 grs de beurre.)

Dans leur tableau IV, il est question de 12 échantillons de beurres provenant de crèmes acidifiées jusqu'à un titre de 74 , puis ramenées par neutralisation partielle au titre de 28 , pasteurisées et barattées. Les titres des distillats étaient de 0,5 à 0,9 .

Leur tableau $\mathrm{V}$ donne les titres des distillats de beurres fabriqués de crèmes très acides, les unes barattées sans pasteurisation, quelques autres après pasteurisation. Le titre le plus bas était de $I, 4$, le plus élevé 3,8 ; les beurres de crèmes pasteurisées donnent ordinairement des titres plus bas que les beurres de crèmes non pasteurisées. (Dans ces 
cas les distillations sont exécutées d'après une méthode qui donne des résultats plus élevés).

Nous conclurons de ces recherches que le beurre fabriqué de crèmes acidifiées contient beaucoup plus d'acides volatils que le beurre fabriqué de crèmes non acidifiées. Au laboratoire, il ne m'était pas possible de fabriquer du beurre avec de la crème non acidifiée et, en conséquence, je n'ai pu déterminer le titre de ces beurres en acides volatils.

J'ai réalisé une approximation en distillant la crème non acidifiée ot en calculant la part qui revenait sur le titre trouvé à la matière grasse contenue dans la crème.

La crème acidifiée pouvant être barattée, les titres des beurres fabriqués avec elles ont montré une bonne concordance avec ceux des beurres achetés ici et là et qui provenaient de crèmes mûries. Pour ces derniers, on ne connaissait rien de la manière dont avaient été acidifiées les crèmes.

6 échantillons de lait écrémé ont donné des titres d'acides volatils de 3,0 à 4,$2 ; 4$ échantillons de crèmes ayant un taux butyreux de $24 \%$, puis pasteurisés à $80^{\circ}$, ont donné des titres de 4,7 à 5,8. Malheureusement, le lait écrémé et la crème ne provenaient pas du même lait et n'étaient pas du même âge. Le lait écrémé provenait d'un lait très fin et très frais, tandis que la crème était âgée, elle avait déjà une odeur désagréable $\mathrm{Si}$, pour le lait écrémé, on suppose un titre moyen de 4 et pour la crème de 5, on aura :

Titre de $250 \mathrm{gr}$. de crème $(24 \%$ de matière grasse $) .. . . . . . .55,0$

Titre de $190 \mathrm{gr}$. de lait écrémé : $4 \times \frac{190}{250}=\ldots \ldots \ldots \ldots . . \ldots 3,0$

Titre de $60 \mathrm{gr}$. de matière grasse : $5,0-3,0=\ldots . . . . .2,0$

Si ce beurre contient $20 \%$ d'eau, 60 gr. de matière grasse correspondent à $75 \mathrm{gr}$. de beurre. Le titre de ces $75 \mathrm{gr}$. de beurre provenant de $250 \mathrm{gr}$. de crème fraîche devient 2,0 et pour $100 \mathrm{gr}$. de beurre 2,7 , valeur qui, d'après ce qui précède, est un peu plus grande que celle du beurre de crème non acidifiée. 3 échantillon. de beurres achetés avaient les titres de $7,5,6,7$ et 5,$5 ; 3$ échantillons de beurres fabriqués par moi de crèmes acidifiées avaient les titres $6,5,8,0$ et 5,7 .

Il résulte que le beurre dissout une partie des acides volatils formés par les bactéries des cultures lactiques employées pour l'acidification de la crème.

\section{BIBLIOGRAPHIE}

[1] B. W. Hammer. New Angles to the Startermakers Problem. - Journal of Dairy Science, 1921 ,

[2] V. Sтовон Fortsatte Undersögelser over Fremstilling af Syverökkere. 102 Beretn. fra Forsögstaboratoriet. Köbenhavn, 1919.

[3] SöNCKE Kundsen. Die Milchsaurebakterien des Sauerteiges. -Den kgl. Véterinoerog Landbohojskoles. Aatsskrift, 1924. 
[4] Virtanen A. J. Bestämning av flyktiga Fettsyvoer i Bakteriekukturer. Medd. No 242 frañ Centralanstalten för försöksväsendet pa jordbrudesomradet, Stocisholm, 1923.

[5] Ferris L. W., ReDfield H. W. et North W. R. The volatil acids and the volatile oxydable substances of cream and experimental butter. - Journal of Dairy Science, 1921.

\title{
LE CONTROLE DE LA PRODUCTION LAITIËRE EN FLANDRE OCCIDENTALE (Belgique)
}

\author{
par Franz VRANKEN. \\ Directeur du Eigenaars et Landbouwersbond, Bruges.
}

Depuis quelques années, le service de Zootechnie du Ministère de l'Agriculture de Belgique s'occupe activement de l'épuration de nos races bovines. Dans ce but, il a désigné pour chaque région du pays la race dont l'élevage présente le plus de chances de succès eu égard au climat, au sol, aux méthodes d'exploitation, etc.

L'établissement du Standard de chaque race rend possible un travail méthodique et facilite la tâche aussi bien des éleveurs que des experts, tandis que la généralisation du contrôle de la production laitière contribue, de son côté, à la découverte des sujets les plus méritants. Enfin, l'organisation des syndicats d'élevage semi-officiels et la création de Herdbooks sérieux permettent d'espérer que, dans un avenir rapproché, la Belgique possèdera un cheptel bovin bien uniforme et de rendement moyen élevé.

Dans la province de la Flandre Occidentale, où la guerre avait complètement anéanti les fameuses étables de la Rouge des Flandres, le relèvement rapide de l'agriculture et de l'élevage avait été rendu possible par l'introduction d'une grande quantité de laitières de provenances les plus diverses. Aussi, dans les premières années qui suivirentl'armistice, le cheptel de cette région était devenu un méli-méloinextricable.

C'est dans cette contrée, et dans le but de seconder les efforts des pouvoirs publics, que l'initiative privée créa une association particulière, nommée Syndicat d'élevage de Wervicq-Gheluwe, dont l'animateur est le distingué Docteur en médecine vétérinaire, M. Paul Vanraes, à Wervicq.

$\mathrm{Au}$ début, ce syndicat s'occupait exclusivement du contrôle des bovidés Dairy-Shorthorn, introduits d'Angleterre, mais plus tard il assuma la tâche de guider les éleveurs de la région dans le choix des races et des types à conserver. Pendant une période de plusieurs années il poursuivit, dans cette intention, le contrôle de la production laitière d'un grand nombre de vaches.

En 1926, ce contrôle a porté sur 284 sujets de huit races différentes subdivisées en quatre catégories d'après le nombre de vêlages.

Voici le tableau des résultats enregistrés : 